Daniela Bernasconi ${ }^{1}$, Robert Steffen ${ }^{1}$ und die Arbeitsgruppe Influenza ${ }^{2}$

1 Institut für Sozial- und Präventivmedizin, Abteilung Epidemiologie und Prävention übertragbarer Krankheiten, Universität Zürich

2 Mitglieder: Jann Cloetta, BAG (Bern), Germain-Marcel Daucourt, Kreisarzt Generalstab (Bern), Urs Hess, BAG (Bern), Daniel Lavanchy, WHO (Genève), Hans C. Matter, BAG (Bern), François Méan, Service de santé publique (Lausanne), A. Schneider, BAG (Bern), Josef Schöpfer, Allgemeinpraktiker FMH (Escholzmatt), Robert Steffen (Präsident), ISPM (Universität Zürich), Dieter Stürchler, BAG (Bern), Urs Thurnherr, Philosophisches Seminar (Universität Basel), Werner Wunderli, NZI (Genf), Hanspeter Zimmermann, BAG (Bern)

\title{
Massnahmen bei einer Influenza-Pandemie \\ in der Schweiz *
}

Zusammenfassung
Da in den nächsten Jahren eine Grippepandemie zu erwarten ist, wer-
den in den Industrienationen Pläne für diesen Fall erstellt, Während in
der interoandemischen Periode die Zustandigkeiten klar geregelt sind
und keine Engpässe bezüglich Impfstoffe und Medikamente bestehen,
ist bei einer Pandemie mit grossem Mangel an Material und Infrastruk-
tur zu rechnen, der gesellschaftlich und wirtschaftlich bedeutende Fol-
gen hat. Der vorliegende Plan skizziert, wie mit den verfügbaren Mitteln
bestmöglich primar die vorzeitige Sterblichkeit gemindert, sekundär die
Morbidität reduziert essentielle Dienste und die Sicherheit des Landes
erhalten werden konnen. Optionen werdeh dargelegt, zB. Wie eine ver-
mehrte Produktion von mpfstoff in der interpandemischen Phase wohl
den pandemiebedingten Schaden vermindern würde.

Der vorliegende Influenza Pandemie-Plan für die Schweiz basiert auf Empfehlungen der 7. Europäischen Konferenz über Influenza und deren Prävention (Berlin $1993)^{1}$. Er ist primär für Personen bestimmt, die im Gesundheitswesen wirken. Auszugsweise soll er im Bulletin des Bundesamtes für Gesundheit (BAG) veröffentlicht werden und soll als Basis für eine entsprechende Verordnung dienen. Grundlage für den Plan ist das Epidemiengesetz (EpG), gemäss welchem „Bund und Kantone treffen auf Grund dieses Gesetzes die nötigen Massnahmen, um übertrag- bare Krankheiten des Menschen zu bekämpfen (Art. 1, Absatz 1).“ „Es (das BAG) gibt zuhanden der Behörden und Ärzte technische Richtlinien zur Bekämpfung übertragbarer Krankheiten heraus und passt sie laufend den wissenschaftlichen Erkenntnissen an (Art. 3 Absatz 3).“

Unter Pandemie versteht man eine zeitlich begrenzte, weltweite, massive Häufung von Fällen einer Infektion. Grippe-Pandemien wie die von 1918 und, in geringerem Ausmass, von 1957 und 1968 (Tabelle 1) treten unter folgenden Bedingungen auf:
- ein bereits zirkulierendes Influenza-Virus hat durch Rekombination (,antigenic shift") ein neues Hämagglutinin- und/oder Neuraminidase-Gen erhalten;

- es besteht bei einem grossen Teil der Weltbevölkerung keine Immunität gegen das neue Virus, und

- das neue Virus breitet sich schnell aus und ist hoch pathogen.

Eine Pandemie ist eine Katastrophe, in welcher wegen Produktionsengpässen mit Mangel an Impfstoff und Insuffizienz der Infrastruktur zu rechnen ist.

Der vorliegende, hier stark gekürzt dargestellte Plan soll im Falle einer Pandemie mit den verfügbaren Mitteln bestmöglich folgende Ziele erreichen:

- Minderung der vorzeitigen Sterblichkeit (Zahl der verlorenen potentiellen Lebensjahre), z.B. durch die Impfung von Personen mit erhöhtem Risiko für Komplikationen, und sekundär die Minderung der totalen Sterblichkeit.

\footnotetext{
* Ein Entwurf, erstellt im Auftrag des Bundesamtes für Gesundheit, Bern.
} 


\begin{tabular}{|c|c|c|c|c|}
\hline Periode & Jahre & $\begin{array}{l}\text { Exzess- } \\
\text { mortalität } \\
\text { (Total) }\end{array}$ & $\begin{array}{l}\text { Jährlicher } \\
\text { Durchschnitt }\end{array}$ & $\begin{array}{l}\text { Rohe } \\
\text { Jahr u } \\
10000\end{array}$ \\
\hline Pandemie & $1918-1920$ & 675000 & 225000 & 218.4 \\
\hline Interpandemie & $1920-1933$ & 368000 & 28338 & 23 \\
\hline Interpandemie & $1933-1957$ & 242000 & 10108 & \\
\hline Pandemie & $1957-1960$ & 115700 & 38567 & 22.0 \\
\hline Interpandemie & $1960-1968$ & 114900 & 14363 & \\
\hline Pandemie & $1968-1972$ & 111927 & 27982 & 13.9 \\
\hline Interpandemie & $1972-1981$ & 198800 & 22089 & 10.3 \\
\hline
\end{tabular}

Tabelle 1. Schätzung der Exzessmortalität durch Influenza in den USA während den Pandemien und in den interpandemischen Perioden, 1918 bis $1981^{2}$.

- Reduktion der Morbidität durch breite Durchimpfung der Bevölkerung mit dem Ziel, die Verbreitung des Virus zu verlangsamen.

- Erhaltung essentieller Dienste wie Gesundheitsversorgung, Transporte, Kommunikation, Information und Versorgung mit Energie, Trinkwasser und Nahrungsmitteln.

- Gewährleistung der inneren und äusseren Sicherheit durch gezielte Impfung der Polizei, Feuerwehr und eventuell von Teilen der Armee.

Die Auswirkungen der Pandemie könnten mit einiger Wahrscheinlichkeit gelindert werden, wenn die öffentliche Hand gemäss Art. 6 EpG einen Impfstoffhersteller verpflichten könnte, alljährlich genügend Vakzine für die Gesamtbevölkerung bereitzustellen. Bei Nichtgebrauch müssten allerdings jährlich 50 Millionen Franken (z.B. für 7000000 Dosen Inflexal $^{\circledR}$ ) abgeschrieben werden. Bei guter Akzeptanz der Impfung gäbe dies den bestmöglichen Schutz, sofern der Impfstoff, der jährlich auf Grund der zu erwartenden Viren neu konzipiert wird, rechtzeitig verfügbar wäre. Da bei zu kurzer Vorwarnzeit der Impfstoff nicht produziert werden kann, wären zusätzlich Medikamente zur Therapie bereitzustellen, was weitere Kosten bereitet. Es ist letztlich ein politischer Entscheid, ob eine derartige medizinisch optimale, aber im internationalen Vergleich kostspielige Lösung gewählt werden soll. Zusätzlich könnte auch die Forschung zwecks Beschleunigung der Impfstoffproduktion oder Entwicklung von neuen Impfstoffen (DNA-Vakzine) gefördert werden.

\section{Interpandemische Periode}

Unter interpandemischer Periode versteht man den Zeitraum zwischen den eigentlichen Pandemien. Summarisch sind die Zuständigkeiten in Anhang 1 dargestellt. Die Surveillance beruht in erster Linie auf dem Sentinella-System, sekundär auf Todesfallstatistiken und der $\mathrm{H}^{+}$(früher VESKA)-Statistik. Zudem melden Laboratorien, speziell auch das Nationale ReferenzZentrum für Influenza (NZI), Genf, ihre Befunde. Die wichtigste Aufgabe des NZI ist die Überwachung der Grippeaktivität anhand der Proben, die von rund $1 / 5$ der Sentinella-Ärzte aus der ganzen Schweiz gesammelt werden.
Alljährlich informiert das BAG in der interpandemischen Phase, um die Bevölkerung, speziell Risikogruppen, bezüglich Influenza zu sensibilisieren und zur Impfung zu motivieren.

In den letzten Jahren standen auf dem schweizerischen Markt jährlich ungefähr 600000 Dosen von Influenza-Impfstoffen zur Verfügung, rund die Hälfte war aus dem Ausland importiert. Dies bedeutet, dass sich üblicherweise weniger als $10 \%$ der Bevölkerung gegen Influenza impfen lässt.

Die Jahresproduktion von Amantadine $\left(\right.$ Symmetrel $\left.{ }^{\circledR}\right)$, des einzigen in der Schweiz verfügbaren Präparates zur Chemoprophylaxe und Therapie gegen Influenza A-Viren beträgt zur Zeit 5000 Packungen à 60 Kapseln; der Lagerbestand beläuft sich durchschnittlich auf 1000 Packungen. Dieses Medikament hat eine garantierte Lagerzeit von 4 Jahren.

Die Firmen GlaxoWellcome und Roche entwickeln zur Zeit in $\mathrm{Zu}$ sammenarbeit mit Biota Holdings respektive Gilead Sciences zwei neue antivirale Medikamente, die mit Amantadin eine Kombinationstherapie ermöglichen werden. Beide Medikamente beruhen auf der Wirkung von sogenannten Neuraminidase-Hemmern, die das Eindringen von Influenza A- und B-Viren in die Zelle blockieren.

\section{Pandemie}

Es ist damit zu rechnen, dass eine Grippepandemie auch in der Schweiz eine schwerste Belastung des Gesundheitswesens bewirkt und zusätzlich gesellschaftlich und wirtschaftlich bedeutende Folgen zeitigt. Einer derartigen Katastrophe entsprechend sind adäquate Vorkehrungen zu treffen, wobei je nach der Bedrohungslage diese mehr oder minder extensiv anzupassen sind. Während den Pandemien 1918 und 1957 in den USA wurde eine Grippe-Inzidenz- 
rate von 30 bzw. $24 \%$ beobachtet ${ }^{2}$; aus diesem Grund schätzt man, dass ca. $1 / 4$ der Bevölkerung erkranken wird. Umgerechnet auf die Schweiz entspricht dies fast 2 Millionen Einwohner. Die Komplikationsrate in Form einer Pneumonie dürfte ungefähr $6 \%$ erreichen $^{3,4}$, d.h. 120000 Patienten. Die Hospitalisationsrate könnte $0.7 \%$ betragen, das entspricht 14000 Einweisungen ${ }^{4}$.

Die Zuständigkeiten sind wiederum aus Anhang 1 ersichtlich.

Es gilt, zum einen Panik zu vermeiden und zum anderen dem Bedürfnis nach sachlicher Information und Offenheit im Umgang mit Problemen der Pandemie gerecht zu werden. Ein allfälliger Konflikt zwischen diesen Zielen soll nach ethischen Prinzipien gelöst werden. Hierbei gilt es, die Bevölkerung zu motivieren, den Anweisungen und Empfehlungen zu folgen. Es ist darauf zu achten, dass die Fragen möglichst vorweggenommen und beantwortet werden und dass nicht reaktiv gehandelt wird. Die Aufgabe des NZI ist es, möglichst früh den pandemischen Stamm in der Schweiz nachzuweisen.

\section{Impfstoffherstellung und -prüfung}

Die gegenwärtige Produktion des SSII beträgt 200000-300000 Dosen/Jahr des trivalenten Impfstoffes. Mit heutigen Herstellungsverfahren vermag das SSII, aus einem Ei maximal 3 Impfdosen à 1 Impfstamm herzustellen (man nimmt an, dass die Pandemie durch ein einziges neues Virus verursacht wird und somit ein monovalenter Impfstoff verwendet werden kann). Der Hämagglutinin-Gehalt des monovalenten Impfstoffes beträgt $15 \mu \mathrm{g}$; der trivalente Impfstoff enthält je $15 \mu \mathrm{g}$ Hämagglutinin der jeweiligen InfluenzaStämme. Bei Bedarf können bis zu 50000 Eier pro Woche verarbeitet werden, was zu einer maximalen Produktion von 150000 Dosen pro
Woche führt. Die Produktion läuft üblicherweise von März bis Juni (14-16 Wochen), d.h. es könnten so in diesem Zeitraum in der Schweiz 2100000 bis 2400000 Impfdosen hergestellt werden. Die Impfstoffherstellung ausserhalb der üblichen Zeitspanne März-Juni wäre möglich; befruchtete Eier sind allerdings nicht das ganze Jahr über in genügenden Mengen erhältlich. Die hiesigen Geflügelproduzenten erfüllen die Qualitätsstandards (Good Manufacturing Practices) der Pharmaindustrie nicht, deshalb werden die befruchteten Eier aus Deutschland und Dänemark bezogen.

Um die Jahrtausendwende wird das SSII die Impfstoffherstellung in Zellkulturen einführen; es ist allerdings noch nicht bekannt, ob und in welchem Ausmass die neue Herstellungsmethode eine Erhöhung der maximalen Produktion bewirken wird.

Der Impfstammlieferant ist das National Institute of Biological Standards and Control in London. Sofern die Pandemie ihren Ursprung in Europa nimmt, was eher unwahrscheinlich ist, wird der benötigte Impfstoff vermutlich nicht rechtzeitig verfügbar sein. Sofern die Pandemie ihren Ursprung wie üblicherweise im Fernen Osten nimmt, so dürften wir eine Vorwarnzeit von einigen Monaten haben, welche die Impfstoffproduktion im geschilderten Ausmass ermöglicht.

Die Impfkampagne wird u.a. der aktuell verfügbaren ImpfdosenMenge angepasst werden müssen. Mit dem aktuellen Produktionsverfahren beträgt die Anzahl Impfdosen bestenfalls 2,4 Millionen (innerhalb 16 Wochen), somit ist ein gravierender Mangel zu erwarten.

Momentan importieren ausländische Firmen jährlich mehrere hunderttausend Dosen InfluenzaImpfstoff in die Schweiz. Es ist zu befürchten, dass diese Quellen im Falle einer Pandemie versiegen, da
Staaten in derartigen Notlagen ein Embargo verfügen. Für diesen Fall ist ein Exportverbot für Impfstoffe oder dessen Vorstufen zu erwägen.

Die Registrierung und die Chargenfreigabe von Impfstoffen und anderen immunbiologischen Erzeugnissen für die Schweiz erfolgt aufgrund der „,Verordnung über die immunbiologischen Erzeugnisse" von 1989. Die Chargenfreigabe bei Impfstoffen dient dazu, die Qualität, Sicherheit und Wirksamkeit der einzelnen Herstellungschargen zu garantieren. Die Registrierung eines Produktes durch die Abteilung Biologika des BAG und die analytische Prüfung jeder Charge sind die Voraussetzungen dafür, dass die Erlaubnis zum Vertrieb für eine spezifische Charge erteilt werden kann. Chargen von lediglich im Ausland zugelassenen Impfstoffen können daher in der Schweiz nicht zum Vertrieb freigegeben werden. Ebenso berechtigt die Chargenfreigabe im Ausland nicht zum Vertrieb in der Schweiz, da bisher keine entsprechenden bilateralen Abkommen unterzeichnet wurden. Ausserdem müssen Änderungen beispielweise in der Herstellung oder der $\mathrm{Zu}$ sammensetzung von Impfstoffen von der Sektion Impfstoffe des BAG genehmigt werden.

Es gibt also drei Prozesse, deren zeitliche Länge im Zusammenhang mit einer Influenza-Pandemie von Bedeutung sind: die Registrierung (12-24 Monate), die Chargenfreigabe (30 Tage) und die Bewilligung von Änderungen (1 Woche bis 6 Monate). Diese Bearbeitungszeiten sind in der Regel notwendig, um die Wirksamkeit, die Sicherheit und die Qualität der in der Schweiz verkauften Impfstoffe sicherzustellen. Selbstverständlich können diese Zeiten durch eine entsprechende Priorisierung reduziert werden. Es muss aber sorgfältig abgewogen werden, wann und in welchem Ausmass die Wirksamkeit, Sicherheit und Qualität beein- 
trächtigt werden. Impfaktionen mit unwirksamen oder schädlichen Impfstoffen könnten fatale Folgen haben.

\section{Impfstrategie}

Erfahrungsgemäss sind nach medizinischen Kriterien folgende traditionelle Gruppen durch Influenza besonders gefährdet und müssten mit dem Ziel der Senkung der Mortalität geimpft werden:

- Erwachsene und Kinder mit chronischen Herz- oder Lungenkrankheiten, die eine regelmässige ärztliche Überwachung benötigen oder im Verlauf des Jahres hospitalisiert waren (darunter sind auch Kinder mit Asthma bronchiale, kongenitalen Herzvitien und zystischer Fibrose);

- Bewohner von Alters- und Pflegeheimen oder von anderen Institutionen für die Pflege von chronisch Kranken;

- Erwachsene und Kinder, die regelmässige ärztliche Betreuung benötigen oder im Verlauf des Jahres hospitalisiert waren wegen chronischen Stoffwechselstörungen (einschliesslich Diabetes mellitus), Niereninsuffizienz, Hämoglobinopathie oder Immunsuppression (einschliesslich Immunsuppression durch Medikamente);

- Kinder und Jugendliche zwischen sechs Monaten und 18 Jahren, die unter langdauernder Aspirintherapie stehen und somit Gefahr laufen, nach einer Influenza an einem Reye-Syndrom zu erkranken;

- Personen $\geq 65$ Jahre;

- Schwangere Frauen. Eine Exzessmortalität bei Schwangeren wegen Influenza wurde während den Pandemien 1918-1919 und 1957 dokumentiert ${ }^{5,6}$. Aus diesem Grund sollte die Impfung wegen dem Komplikationsrisiko im dritten Trimester in Betracht gezogen werden und dies auch für Frauen, die keiner Risikogruppe angehören.
Die AG-Influ wird, basierend auf den medizinischen Kriterien, eine Prioritätenliste erstellen im Sinne eines Vorschlages zuhanden des Krisenstabes (Anhang 2).

Um die gesellschaftlichen und politischen Folgen der Pandemie zu reduzieren, müssen zusätzlich folgende Impfstoffempfänger erwogen werden:

- Personal des Gesundheitswesens: in Spitälern, Arztpraxen, Spitexeinrichtungen, Alters- und Pflegeheimen;

- Angestellte der Transportbranche (im Personentransport, aber auch Lebensmittel- und Medikamententransport);

- Angestellte der Energie- und Wasserversorgung;

- Personal in der Nachrichtenübermittlung;

- politische Entscheidungsträgerinnen und Entscheidungsträger.

Es ist Aufgabe des Krisenstabes, eine Prioritätenliste zu erstellen, die die medizinische und die gesellschaftlich-politische Ebene zugleich berücksichtigt bzw. beide Ebenen zusammenführt. Diese Prioritätenliste wird der Lage entsprechend laufend modifiziert. Dabei werden in Betracht gezogen: die Menge an verfügbarem Impfstoff, die Charakteristika des pandemischen Virus und die ethischen Gesichtspunkte. Es ist denkbar, dass eine bestehende Teilimmunität (errungen im Verlauf früherer Influenza-Epidemien) unterschiedliche Inzidenz- und Mortalitätsraten in unterschiedlichen $\mathrm{Al}$ tersklassen verursacht. Dieses Phänomen wurde während der Hong Kong Pandemie von 1968 beobachtet; damals wies die Altersgruppe der über 80 jährigen eine geringere Exzessmortalität als die Gruppe der 70- bis 79jährigen auf 7,8 .

Die endgültige Entscheidung über Prioritäten bezüglich Gruppen von Impfstoffempfängern obliegt den politischen Instanzen (der Bundesrat auf Antrag der AG-Influ beziehungsweise des Krisenstabes).
Gemäss dem EpG (Art. 23 Abs. 2) bestünde die Möglichkeit der Zwangsimpfung: „Die Kantone bestimmen, ob diese Impfungen freiwillig oder obligatorisch sind". Theoretisch könnte man dies in Betracht ziehen, um die anzustrebende Herdimmunität (man schätzt, dass die erforderliche Durchimpfungsrate bei $75 \%$ liegt ${ }^{9}$ ) zu fördern. Das EpG würde weitere Zwangsmassnahmen zur Eindämmung einer Epidemie vorsehen, wie die ärztliche Überwachung (Art. 15) und die Absonderung (Art. 16). Solche Massnahmen sind aber im Fall von Influenza nicht sinnvoll, weil eine Einschränkung der Freizügigkeit eine Grippepandemie nicht wirksam verhindern kann.

Impfstoffverteilung während der Pandemie

Für die Verteilung der Vakzine während einer Pandemie sollten die bestehenden Kanäle (vom Impfstoffhersteller zu den Grossisten und den Ärzten bzw. Apotheken) soweit möglich benutzt werden. Um die knappe Menge an Impfstoff zu Beginn der Impfkampagne optimal einzusetzen, werden die Impfaktionen nur an ausgewählten Stellen durchgeführt; mit deren Belieferung ist die Firma (SSII) oder eventuell ein Grossist zu beauftragen. Die kantonalen Krisenstäbe bezeichnen die Impfstellen; als Kandidaten kommen in erster Linie Krankenhäuser und Schulen, bei Bedarf auch die Krankenabteilungen der Waffenplätze in Frage.

Anwendung von Amantadin zur Prophylaxe und Therapie von Influenza-A-Erkrankungen

Der wichtigste limitierende Faktor (neben den Schwierigkeiten bei der Dosierung für bestimmte $\mathrm{Pa}$ tienten) in der Planung der (Massen-)Anwendung liegt in der geringen Produktion von Amantadin. 
Der Lagerbestand beläuft sich auf durchschnittlich 1000 Packungen; Geigy Pharma könnte (mit einer Latenz von 8-12 Wochen) zusätzliche 5000 Packungen liefern. Zur Erinnerung: eine Packung enthält 60 Kapseln à $100 \mathrm{mg}$. Mit 6000 Packungen könnte man 12000 Erwachsene (14-64 Jahre) eine 15tägige Prophylaxe (à $200 \mathrm{mg} / \mathrm{Tag}$ ) verschreiben; mit derselben Menge könnten 24000 Erwachsene im Alter von $\geq 65$ Jahren (à $100 \mathrm{mg} /$ Tag) eine 15tägige Prophylaxe erhalten. Zielgruppen einer Prophylaxe wären vor allem Personen mit einer Neigung zu Komplikationen bei einer Influenza A-Infektion, die nicht geimpft werden konnten. Eine Packung des Medikaments kostet 40.- SFr.; die Gesamtkosten der Prophylaxe können wie folgt berechnet werden: 6000 Packungen à 40.- $=240000 .-$ SFr.

Die Anwendung von Amantadin in der Therapie der Grippe würde eine punktuelle Hilfe für Betroffene darstellen und ein Manko an Impfstoff nur geringfügig kompensieren.

Vorbereitung auf die "second wave"

Nach Abklingen der ersten Pandemiewelle sollte man die Impfung von Bevölkerungsgruppen vorbereiten, die während der ersten Impfkampagne nicht berücksichtigt werden konnten. Zusätzlich muss die Surveillance erhöht werden, um eine erneute Zunahme der Erkrankungen und das Wiederauftreten des Virus rechtzeitig zu entdecken. Diese sogenannte "second wave" kann ähnliche Auswirkungen wie die erste Pandemiewelle haben.

Gesellschaftliche Massnahmen/ Behavior modification

Das Tragen von Gesichtsmasken als individueller Schutz (wie z.B. das Modell 1814 der Firma 3M, ein sogennanter „Respirator", der zum
Schutz des medizinischen Personals bei der Pflege von Tuberkulose-Patienten gebraucht wird) kann nicht empfohlen werden.

Es liegen nämlich keine unumstrittenen Wirksamkeitstests vor, die mit lebenden oder abgetöteten Keimen durchgeführt worden sind (die Masken werden mit Latexpartikeln getestet). Die Filterwirkung einer Atemschutzmaske bleibt solange erhalten, bis die Maske durchfeuchtet oder mit Partikeln verstopft ist. Dies erfolgt normalerweise innerhalb von 2 bis 3 Stunden. Solche Massnahmen würden zusätzliche Kosten verur- sachen und die Träger in falscher Sicherheit wiegen.

Während der Pandemie ist die Bevölkerung über Expositionsprophylaxe $\mathrm{zu}$ informieren; darunter zählt (soweit möglich) die Vermeidung von grösseren Menschenansammlungen (Benutzung der öffentlichen Verkehrsmittel in Stosszeiten, Restaurant- und Kinobesuche etc.). Weiter sind Angaben über Selbsttherapie mittels Hausapotheke zu verbreiten, wobei bezüglich Medikamente stets auch über deren potentielle $\mathrm{Ne}$ benwirkungen und Kontraindikationen zu informieren ist ${ }^{10}$.

\section{Summary \\ Contingency plan in case of an influenza pandemic in Switzerland}

As an influenza pandemic is to be expected, contingency plans are being developed in industrialized nations. During the interpandemic phase the responsabilities are clearly defined and no shortage occurs with respect to vaccines and drugs. In contrast, a lack of material and of infrastructure must be expected in a pandemic which will stress public health and disrupt social and business life. This plan illustrates how with the available means we should attempt to the best possible primarily to reduce premature mortality, secondarily to diminish morbidity and to maintain essential public services and security. Options are described, for instance how an increased production of vaccine already during the interpandemic phase would probably reduce the impact of a pandemic.

\section{Résumé}

\section{Mesures à prendre lors de pandémie grippale en Suisse}

Il faut compter avec une pandemie grippale au cours des prochaine années, raison pour laquelle les nations industrialisées préparent des mesures pour faire face à cet événement. En période interpandémique les compétences sont clairement réglées et vaccins ou médicaments sont disponibles en quantités suffisantes. Lors de pandémie il faut au contraire compter avec des insuffisances importantes tant quant au matériel qu'à l'infrastructure, insuffisances qui auront des conséquences économiques et sociales considérables. Les présent projet esquisse comment I'on peut, au mieux et avec les moyens disponibles, en premier lieu réduire la mortalité prématurée et, en deuxième lieu, réduire la morbidité et maintenir les services essentiels et la sécurité du pays. Des options sont présentées, par exemple, comment une production accrue de vaccin déjà pendant la phase interpandémique réduirait l'impact de la pandémie 


\section{Literaturverzeichnis}

1 Recommendations of the 7th European Meeting of Influenza and Its Prevention. Eur J Epidemiol 1994, 10:525-526.

2 Glezen $P W$. Emerging infections: Pandemic Influenza. Epidemiologic Reviews 1996; 18:64-76.

3 Collins SD. Age and sex incidence of influenza and pneumonia morbidity and mortality in the epidemic of 1928-1929 with comparative data for the epidemic of 1918-1919. Public Health Reports 1931; 46: 1909-1937.

4 Sentinella 1992/93. Ergebnisse des Erhebungsjahres Juni 1992 bis Dezember 1993. BAG, 1995.

5 Philit $F$, Cordier JF. Therapeutic approaches of clinicians to pande-

\section{Anhang 1}

Kompetenzen, Ablauf der Interventionen

Im Folgenden werden die Aktivitäten vorgestellt, die im Fall einer Influenza-Pandemie in der Schweiz aufgenommen werden könnten. Die Interventionen werden der Lage entsprechend angepasst.

\section{Phase 0: Interpandemie}

BAG:

- Surveillance (Sentinella)

- Information (collection and dissemination)

- Analytische Prüfung und Registrierung von Impfstoffen

- Erarbeitung und Veröffentlichung der Empfehlungen zur Grippeprävention; Erhöhung der Durchimpfungsrate, Propagierung des Impfstoffes

- Vorbereitung des Pandemieplanes mit der AG-Influ

- Erstellung einer Verteilungsliste für den Pandemieplan

NZI:

- Isoliert und identifiziert die zirkulierenden Influenza-Viren ganzjährig mic influenza. Eur $\mathrm{J}$ Epidemiol 1994; 10:491-492.

6 Harris $J W$. Influenza occurring in pregnant women. JAMA 1919; 72, 978-980.

7 Egger M, Jennings S, Spuhler Th, Zimmermann HP, Paccaud $F$, Somaini B. Sterblichkeit während Grippeepidemien in der Schweiz 1969-1985. Schweiz Med Wschr 1989; 119:434-439.

8 Housworth W, Spoon MM. The age distribution of excess mortality during A2 Hong Kong influenza epidemics compared with earlier A2 outbreaks. Am J Epidemiol 1971; 94:348-350.

9 Selby $P$ (ed). Influenza: Virus, Vaccines, and Strategy. Proceedings of a working group on Pandemic Influenza, Rougemont, 26-28

- Stellt die Resultate in einem Bericht vor

- Information

AG- Influ:

- Vorbereitung und Aktualisierung des Pandemieplanes

- Empfehlungen für die Grippeprävention mit der SKIF

Kantone:

- Umsetzung der Empfehlungen für die Grippeprävention des BAG

- Erfassung der Durchimpfung

- Erhöhung der Durchimpfungsrate und Propagierung des Impfstoffes

SSII:

- Herstellung des Influenza-Impfstoffes nach den Richtlinien der WHO

Phase 1: Entdeckung eines Influenzavirus mit neuem HA-und/oder NA-Gen

BAG:

- Intensivierte Datensammlung durch Kontakte zur WHO; wie-
January 1976. Academic Press 1976.

10 Patriarca PA, Cox NJ. Influenza Pandemic Preparedness Plan for the United States. The Journal of Infectious Diseases 1997; 76 (Suppl 1): S4-7.

\section{Korrespondenzadresse}

Prof. Dr. Robert Steffen

Institut für Sozial- und

Präventivmedizin

der Universität Zürich

Sumatrastrasse 30

CH-8006 Zürich

Fax +41/1634 46984

travclin@ifspm.unizh.ch viele Fälle und wo, mit Angaben zu Altersklassen, Hospitalisationsraten, Letalität, Geschwindigkeit der Ausbreitung,

- informiert die Kantonsärzte und die Ärzteschaft,

- konsultiert die FMH bezüglich den Präventionsmassnahmen

NZI:

- Beschaffung der Reagenzien zur Isolation und Typisierung des neuen Virus

- Datenbeschaffung über die Eigenschaften des neuen Virus und deren Empfindlichkeit auf Antivirals durch Kontakte zur WHO in Genf, zu den World Collaborating Influenza Centres der WHO und weiteren Expertengruppen aus dem Ausland

AG-Influ:

- Evaluation der Situation anhand der ersten Informationen

- Beratung des BAG

- Erarbeitung eines Vorschlages für die Impfstrategie (zuhanden des Krisenstabes während Phase 2) 
SSII:

- Vorbereitungen für eine allfällige neue Impfstoffproduktion

Falls das neue Virus kein pandemisches Potential hat: zurück zu Phase 0.

Phase 2: Pandemie noch ausserhalb der Schweiz

WHO:

- die Task Force deklariert die Pandemiebedrohung

Bundesrat:

- bestätigt die Pandemiebedrohung

- aktiviert Teile des KSD

- entscheidet über Gruppen von Impfempfängern auf Antrag der AG-Influ beziehungsweise des Krisenstabes

BAG:

- setzt gemäss Pandemieplan einen Krisenstab ein

- die Sentinella-Ärzte erhöhen die Zahl der Abstriche

- setzt einen Informationsstab für die Bevölkerung ein

- mit der AG-Influ berät und unterstützt den Bundesrat bei der Erklärung der Pandemiebedrohung und zur Impfstrategie

Krisenstab:

- Erstellung einer Impfstrategie nach gesellschaftlich-politischen und medizinischen Kriterien zuhanden des Bundesrates

- Erarbeitung eines der Situation angepassten Massnahmenpaketes zur Implementation der beschlossenen Impfstrategie

- Koordination der Massnahmen der Kantone

- logistische Organisation der Impfstoff- und Antiviralsverteilung mit dem KSD und dem SSII

Kantone:

- aktivieren Teile der kantonalen Krisenstäbe

- Bereitstellung der medizinischen Infrastruktur

- Organisation der Verteilung des Impfstoffes und Information der Bevölkerung
NZI:

- erhöht die Zahl der Isolationen und Typisierungen

- Einspannung und Koordination der Tätigkeit aller DiagnostikLaboratorien

- Analyse einer Serothek zur Bestimmung von Risikogruppen

SSII:

- stellt den adäquaten Impfstoff her und bestimmt deren Immunogenität

- organisiert die Verträglichkeitsstudien

- sobald möglich verteilt den Impfstoff an die Kantone

\section{Phase 3: Pandemie in der Schweiz}

Bundesrat:

- deklariert die Pandemie in der Schweiz

BAG:

- setzt die Surveillance fort

- mit der AG-Influ berät und unterstützt den Bundesrat bei der Entscheidung über Massnahmen für die Eindämmung der Pandemie

Krisenstab:

- setzt die Massnahmenpakete mit den Kantonen um

- ist mit den kantonalen Krisenstäben ständig in Kontakt und erhält Informationen über die Lage und allfällige Schwierigkeiten

Kantone:

- verteilen den Impfstoff in Zusammenarbeit mit dem KSD und dem Hersteller

- führen die Impfaktionen durch

- gewährleisten die medizinische Versorgung

- regeln die Patientenaufnahme und den Patiententransport

- berichten dem Krisenstab über die Lage

NZI:

- setzt die Virusisolationen in angemessener Zahl fort

- koordiniert und sammelt die Daten aller Laboratorien
- wird die Surveillance bis zum definitiven Abklingen der Pandemie oder zu Beginn der "second wave" fortsetzen

SSII:

- stellt den Impfstoff her

\section{Phase 4: Ende der Pandemie}

Bundesrat:

- deklariert Ende der Pandemie

BAG, AG-Influ, NZI, Krisenstab, KSD, SSII:

- Auswertung aller Daten zur Pandemie und Evaluation aller Interventionen; Erstellung eines Berichtes über die Pandemie in der Schweiz zuhanden des Bundesrates.

\section{Anhang 2}

Die Impfkampagne an einem hypothetischen Beispiel

Anlehnung an die Bedingungen, die 1968 in den USA herrschten:

- Neues Virus in Hong Kong Mitte Juli entdeckt (Tag 0)

- 120 Tage bis zur Freigabe der ersten Dosen in den USA (zirka Mitte November)

- Beginn der Epidemie in den USA im Dezember, in der Schweiz im Januar

Für die Impfkampagne stehen rund 6 Wochen bis zum Ausbruch der Pandemie zur Verfügung, falls der Impfstoff in 4 Monaten hergestellt und freigegeben wird; der Beginn der Impfkampagne deckt sich mit dem Beginn der Pandemie in der Schweiz, falls 6 Monate erforderlich sind.

Mögliche Impfstrategien:

a. Die Priorität wird Personen in den Risikogruppen gegeben, sekundär werden Angehörige beruflich gefährdeter und bedeutsamer Gruppen geimpft.

b. Die Vertreter der wichtigsten Berufsgruppen werden prioritär geimpft, Personen mit Risikofaktoren in einer zweiten Phase. 
Ablauf der Impfkampagne am Beispiel der Strategie b:

\section{Woche}

Spitäler 110000

Arztpraxen 27000

Andere Krankenschwestern/ -pfleger

Medizinische Labors

Total

148000

2. Woche

Alters- und Pflegeheime

Apotheken

12000 Beginn der Impfkampagne

Hersteller von Impfstoffen, Antibiotika und anderer wichtiger Medikamente Ambulatorien 8000

3000

40000

Grosshandel für

Pharmazeutika

Nachrichtenübermittlung

Wasser-, Energie-

versorgung

Total

3. Woche

Wasser-, Energie-

versorgung

Transportwesen

10000

140000

4. Woche

für die Allgemeinbevölkerung

Politische Entschei-

4000 dungsträger

1600

5200

71000

17000

150800

Polizei/Feuerwehr
Dauer der Impfkampagne bei 150000 Dosen/Woche für

- chronisch Kranke aller Altersgruppen: 6 Wochen (900000 Personen),

- Personen $\geq 65$ Jahre: 7 Wochen (1044000 Personen),

- Schulkinder: 7 Wochen (1053000 Personen),

- die ganze Bevölkerung: 46 Wochen (7000000 Personen).

Unter ungünstigen Bedingungen (keine Eier verfügbar oder eine zu kurze Zeitspanne für die Impfstoffproduktion) wird kein Impfstoff zur Verfügung stehen. 